Radiologe 2011·51:9

DOI 10.1007/s00117-010-2082-x

Online publiziert: 24. November 2010

๑) Springer-Verlag 2010

D. Theisen · M.F. Reiser

Institut für Klinische Radiologie, Klinikum der

Ludwig-Maximilians-Universität München, Campus Großhadern, München

\title{
MRT und CT bei angeborenen Herzfehlern
}

lung der zumeist komplexen anatomischen Verhältnisse, eine exakte und gut reproduzierbare Quantifizierung der Ventrikelfunktion sowie, als einziges nichtinvasives Verfahren, die Messung des Blutflusses. Dadurch hat die MRT eine große Bedeutung als komplementäres Verfahren zur Echokardiographie in der Primärdiagnostik erlangt. Sie wird immer dann angewendet, wenn kein ausreichendes Schallfenster zur Echokardiographie vorhanden ist oder diese nicht alle therapieentscheidenden Informationen liefern kann. Klassische MR-Indikationen stellen die komplexen Herzfehler dar, bei denen es neben der Darstellung der intrakardialen Anatomie und Funktion auch um eine Beurteilung der großen intrathorakalen Gefäße geht. Gutberlet et al. erläutern die Strategie der Bildgebung bei der Transposition der großen Gefäße. Der Beitrag von Wintersperger et al. zeigt die Stärken der MRT bei der Therapiekontrolle von Patienten mit Aortenisthmusstenose auf.

Die MRT kann aber auch bei scheinbar weniger komplexen Herzfehlern wie Vorhof- und Ventrikelseptumdefekten wichtige Zusatzinformationen liefern. Huber et al. beschreiben in ihrem Beitrag, wie man die MRT gezielt zur Therapieplanung bei Patienten mit Vorhofseptumdefekt einsetzen kann.

Durch den Einsatz innovativer, dosissparender Untersuchungsprotokolle bei Geräten der neuesten Generation findet auch die Computertomographie zunehmend Eingang in die Abklärung angeborener Herzfehler. Leschka et al. machen uns mit den Indikationen und aktuellen Protokollen vertraut.
Wir hoffen, mit diesem Themenheft einen verständlichen und für die klinische Praxis nützlichen Überblick über den Stand der Entwicklung auf dem Gebiet der kardiopulmonalen Bildgebung bei angeborenen Herzfehlern zu geben, der Ihr Interesse an diesem spannenden Thema wecken kann.

Ihre

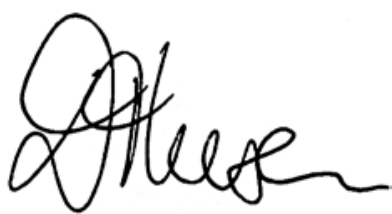

Dr. Daniel Theisen

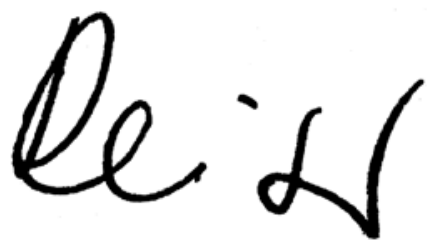

Prof. Dr. Dr. h.c. Maximilian F. Reiser 